\title{
Flashes in femtoseconds
}

For the molecule, time ticks in picoseconds, or even femtoseconds - respectively $10^{-12}$ and $10^{-15} \mathrm{~s}$. On such timescales, a molecule tumbles through space, an atom vibrates back and forth in a crystal, a chemical bond is forged or broken. Yet the development of pulsed lasers blinking in a femtosecond staccato of flashes has enabled these fundamental molecular processes to be charted, revealing the rise and decline of ephemeral intermediates under the furiously strobed illumination.

Femtosecond spectroscopy has shown us the dynamics of bond breaking — but what of the structural aspects? When will we be able to map out the coordinates of individual atoms, as, for example, a protein docks onto the cell surface or as a chlorine radical eliminates an ozone molecule? When will we see the first atomically resolved diffraction pattern of a true transition state, and watch frame by frame as

vibrations carry atoms into new unions?

This demands a marriage of the techniques of ultrafast spectroscopy with those of X-ray diffraction. The probe beam becomes a pulsed X-ray source, its flashes brief enough to 'freeze' the atomic motions yet bright enough to provide a discernible diffraction pattern. Current synchrotron sources can be pulsed at around 50 picoseconds, whereas ultrashort laser pulses can excite bursts of X-rays from suitable targets that could, in principle, be over within 100 femtoseconds.

These pulses must be synchronized with an optical pumping beam, which gets the reaction underway. An alternative is to diffract electrons rather than X-rays. Diffraction on the 'molecular' timescale of femtoseconds is an infant discipline which promises wonders once perfected, but which is capable right now of only the crudest of impressionistic sketches: blurred images of lattice dynamics, showing evidence of rapid change but without a single molecule (let alone an atom) in focus. The static photography of the Braggs has yet to produce its first movie. Philip Ball

\section{Arrhythmias}

'His heart raced'. 'Her heart skipped a beat'. 'My heart beat sluggishly in my breast.' Literature is peppered with such lines. Why? Because, as many a dramatic writer has noted, a disturbance in that most basic 'rhythm of life' — the normal human heart rate of between 60 and 100 beats per minute — is a sign that something is amiss.

Normally a heartbeat starts in the right atrium of the heart, in a specialized group of pacemaker cells known as the sinus node. This node sends an electrical signal, which spreads through the atria and ventricles of the heart, following a precise route that induces the heart to beat in the most efficient way.

If the sinus node develops an abnormal rate or rhythm, if the conduction pathway of its electrical signals is faulty or if another part of the heart takes over as the pacemaker, arrhythmias - as the various types of irregular heartbeat are generically known - can ensue. Arrhythmias may be due to heart disease, ageing, medications, metabolic imbalances or other genetic or infectious medical problems. And the ineffective blood pumping that they give rise to can cause fatigue, dizziness, light-headedness, fainting spells and, ultimately, strokes and death.

Oscillations in the cells of the sinus node operate by setting up a cyclic decay of the difference between the charge on the outside of the membrane and the inside. This causes the membrane to fire spontaneously, which raises its conductance and allows electrolytes to rush in, thus reversing the potential across the membrane. This reversed charge propagates to neighbouring cells, causing the same sequence of events therein and hence a concert of muscular contraction.

But it is currently unclear exactly how cyclically expressed genes and proteins give rise to cyclic decay in membrane potential in the heart's muscle cells. So, just as in many other areas of medical biology, the challenge now is twofold. First, to expand our knowledge of the genetics and cell signalling processes that gives rise to healthy heartbeats. And second, to integrate this into a far richer picture of the workings, and failings, of the whole heart.

Sara Abdulla

\section{Does the past have a future?}

Hardly a week goes by without the discovery of some amazing fossil that will change the way we look at the history of life. But how Iong can the fossil bonanza last? After all, the Earth has only so many rocks to investigate. Will there come a time when we can say that the fossil record is substantially complete, in that the likelihood of discovering radically new forms, or significant range extensions of known forms, becomes improbable? If so, when will that time arrive? And when it does, how will this influence the practice of palaeontologists?

"I think we are more or less at that point now," says Andrew Smith of the Natural History Museum in London. "The available outcrops are pretty heavily searched within the developed nations, and major headway has been made in exploring places like Mongolia and Madagascar." His prognosis is, however, tempered by the fact that absolute completeness is an impossibility, because the vast majority of organisms do not end up as fossils: "my belief is that we will have a substantially biased fossil record that cannot be bettered no matter where we go in the world".

Charles Marshall of Harvard University agrees that the Earth's fossil riches have been fairly well worked out, but says that one cannot discern a point when the last fossil will have been extracted. "One can't simply extrapolate from what has been discovered because radical new finds often result from unanticipated ways of looking, often in stratigraphic intervals or rock types not typically examined. If the rate of recent discoveries, from fossil embryos to downcovered dinosaurs, are any indication there is much to be discovered yet!"

However, contrary to popular iconography, most palaeontologists do not devote most of their time to making radical discoveries. Such discoveries, says Marshall, will be - as they are now "largely the unexpected by-products of mature research programmes designed to establish the patterns of evolution and elucidate the processes responsible for those patterns."

"The obvious priority," says Smith, "will be to establish and understand how sequence stratigraphy and sea-level change control facies preservation, and thus control apparent biotic diversity and extinction patterns through time. Only then will we begin to get a reliable handle on how diversity has changed over time."

Henry Gee 